\title{
Static electrification by asymmetric rubbing
}

\section{$\operatorname{AUTHOR}(S)$ :}

Komatsu, T. S.; Hashimoto, M.; Miura, T.; Arakawa, I.; Nasuno, $\mathrm{S}$.

\section{CITATION:}

Komatsu, T. S. ... [et al]. Static electrification by asymmetric rubbing. 物 性研究 2004, 81(6): 896-899

ISSUE DATE:

2004-03-20

URL:

http://hdl.handle.net/2433/97763

RIGHT: 


\title{
Static electrification by asymmetric rubbing
}

\author{
T. S. Komatsu, M. Hashimoto, T. Miura, I. Arakawa, and the late S. Nasuno \\ Dep. of Phys., Gakushuin Univ. Mejiro 1-5-1, Tokyo 171-8588, JAPAN
}

\begin{abstract}
We investigate static electrification due to asymmetric rubbing. Eight different plastic materials, for which self-consistent electrostatic series can be obtained, are investigated here. Two rotating disc samples whose edges brought into contact are rubbed each other. The surface voltages of the rubbed surfaces are measured systematically changing the degree of asymmetry of the rubbing by varying the rotation speeds of the two discs. It is found that the tendency of the charge separation depends on the species of materials. This might indicate there are two or more mechanism of charge separation.
\end{abstract}

$46.55 .+\mathrm{d} 81.90 .+\mathrm{c} 81.40 . \mathrm{Pq}$

\section{INTRODUCTION}

Static electricity is a common and unavoidable phenomenon in our daily life. In today's industrial system, anti-static treatment is one of important processes. Nevertheless, the phenomenon has not yet been fully understood. In many occasion, static electrification occurs between two different materials, and such cases have been elaborately investigated. ([1-5]) When two different materials are brought into contact, the separation of charge occurs. It is known that a self-consistent electrostatic series can well describe the sign of charge separation for a given combination of materials. Nevertheless electrostatic series can say nothing about the case two nominally identical surfaces are brought into contact. Although it is known that the separation of charge will occur when two identical surfaces are rubbed asymmetrically ([6]), it seems still unclear how it separates and why it separates. Thus it would be interesting to investigate what is really observed when two identical materials brought into contact.

As a first step to clarify these problems, we made an apparatus to measure static electrification generated by asymmetric rubbing. The apparatus has two rotating discs whose edges are brought into contact and rubbed with each other. The surface voltages of the rubbed surfaces were measured systematically changing the degree of asymmetry of the rubbing by varying ratating speed of two discs. We here present observed tendency of charge separation, which depends on the species of materials. These observations might shed light on dynamical aspects of static electrification and contribute to a discovery of a new secret in nonequilibrium phenomena.

\section{EXPERIMENTAL METHOD}

Eight kinds of plastic materials listed in Table 1. were investigated in this paper. Each sample was prepared in a disk shape with radius $70 \mathrm{~mm} \phi$ and thickness $5 \mathrm{~mm}$. The peripheral edge of the disk had a round shape with a curvature radius $5-10 \mathrm{~mm}$ in perpendicular direction

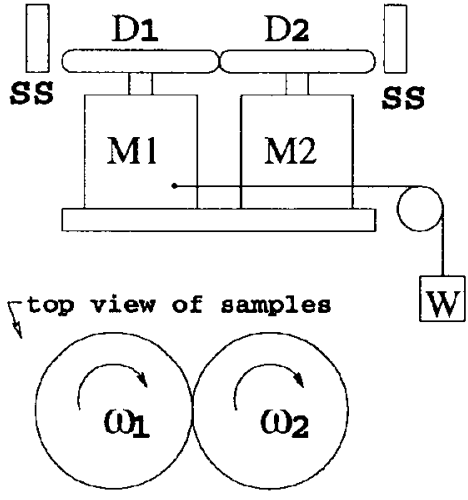

FIG. 1: Experimental apparatus. Samples $\left(D_{1}, D_{2}\right)$ are mounted on stepping motors $\left(\mathrm{M}_{1}, \mathrm{M}_{2}\right)$, brought into contact using a weight $(\mathrm{W})$, and rotated with speed $\left(\omega_{1}, \omega_{2}\right)$. Surface voltage is measured using surface electric potential sensor (SS).

so that two disks could make a point contact when those peripheral edges were brought into contact. The surface of the peripheral was polished with sandpaper (\#3000) or rubbing compound and rinsed in ultrasonic cleaner. Schematic figure of our experimental apparatus is shown in Fig 1. Two stepping motors were mounted on a base plate: one of which $\left(\mathrm{M}_{1}\right)$ could smoothly slide toward the other one $\left(\mathrm{M}_{2}\right)$ and was pulled at a constant force $(0.5-1 \mathrm{~N})$ by a bullet weight $(\mathrm{W})$ while $\mathrm{M}_{2}$ was fixed on a bottom plate. Two disk samples $\left(D_{1}\right.$ and $\left.D_{2}\right)$ were mounted on these motors and rotated independently with an arbitrary chosen speed. Varying the ratio of the rotating speeds of two disks, we can change the degree of asymmetry of rubbing.

Here, the sign of rotating speed is defined as positive in clockwise direction and the rotating speeds of $D_{1}$ and $D_{2}$ are denoted by $\omega_{1}$ and $\omega_{2}$, respectively. Note that symmetric rolling friction occurs when $\omega_{1}=-\omega_{2}$, and that symmetric sliding friction occurs when $\omega_{1}=\omega_{2}$.

Charge appeared on peripherals was measured by the surface electric potential sensors (SS) placed beside the peripherals with separation distance $1.5-2 \mathrm{~mm}$ (corresponding spatial resolution were $2-5 \mathrm{~mm} \phi$ ), and the dis- 


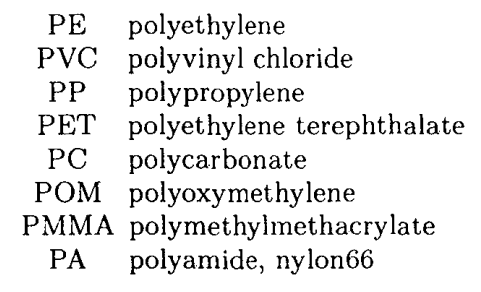

TABLE I: Electrostatic series. After symmetric rolling friction with any combination of (different) materials, upper one has negative charge and lower one has positive charge.

tribution of the surface voltage along the peripheral was obtained.

Whole apparatus were installed in a vacuum bell jar, and some experiments were carried out in Nitrogen gas $(50 \mathrm{kPa})$ environment while the other were performed in normal atmospheric air environment. In both the conditions, similar results were obtained and no noticeable differences have been so far observed except that diffusion of charge seems larger in normal air.

For initial condition of the sample, we prepared almost discharged states (surface voltage at most $\pm 20 \mathrm{~V}$ ) by carefully cleaning the surface of the sample with ethylalcohol or by spraying ionized air.

\section{ELECTRIFICATION INDUCED BETWEEN DIFFERENT MATERIALS}

At first, we investigated electrostatic series of our eight materials. For all combinations of eight materials, samples were brought into contact and rotated with $\omega_{1}=-\omega_{2}=6 \mathrm{rpm}$ (symmetric rolling friction). As a result, we obtained a self-consistent electrostatic series as shown in Table 1. The observed surface voltage were around $\pm 1-4 \mathrm{kV}$ except for samples POM and PA. These samples had relatively small surface resistances, and charge escaped to the ground within minutes. The result of electrostatic series was robust and the order of materials is roughly consistent with the previous works $([7])$.

\section{ELECTRIFICATION INDUCED BETWEEN IDENTICAL MATERIALS}

Let us focus on the cases with identical pairs. Surface voltage after asymmetric rubbing of $\mathrm{PP}$ is shown in Fig. 2 . The samples were brought into contact and rubbed with $\left(\omega_{1}=0.012, \omega_{2}=-0.12 \mathrm{rpm}\right)$ in the first half duration and then with $\left(\omega_{1}=0.12, \omega_{2}=-0.012 \mathrm{rpm}\right)$, so each disk made 0.9 round roll interchanging the asymmetry of the rubbing from $1: 10$ to $10: 1$ at the mid time. In Fig. 2 , it is noticed there are two plus peaks of the surface voltage. These peaks are just located on the surfaces of the slower rotating disks. (Note the slower rotating disk
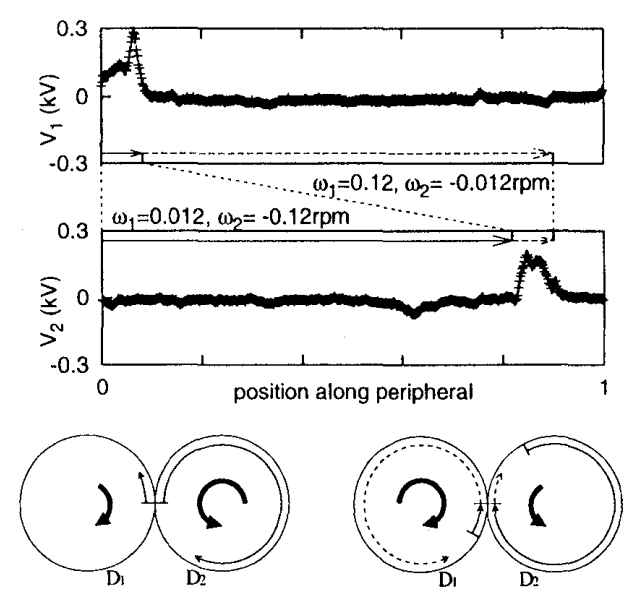

FIG. 2: One snapshot of the surface voltages $V_{1}$ and $V_{2}$ along the peripherals after asymmetric rubbing of PP. Horizontal axes represent positions along the peripherals (normalized by peripheral length) and their origins are set to initial contact positions and they are aligned in contact order from 0 to 1 . In other words, the direction of the rotation is taken as negative for each sample. In undermost schematic figures, thick arrows represent direction of rotation and thin arrows represent coordinates along the peripherals. In the first half duration (left), $D_{1}$ and $D_{2}$ were rotated with $\left(\omega_{1}=-\omega_{2} / 10=0.012 \mathrm{rpm}\right)$, and then the speeds were changed (right) to $\left(\omega_{1}=-10 \omega_{2}=0.12 \mathrm{rpm}\right)$.

is $D_{1}$ in first half duration and then $D_{2}$.) On the other hand, on the faster disk, small negative charge appears. These observations would result from a static electrification due to asymmetric rubbing. In most cases, on a slower rotating disk (SRD), larger magnitude of the surface voltage was observed. It is reasonable because the surface of SRD come into contact with broader area of the other disk.

Typical observations for several materials are shown in Figs.2 and 3. For PP and PET materials, slower rotating disk (SRD) tended to have positive charge but there were also some exceptional observations. For PVC, in many observations, SRD tended to have negative charge but we noticed that the polarity was not robust. For example, it was observed the polarity altered after many revolutions with several manners of contact. It was also observed that SRD had positive charge once after the usage of compounds for preparation and SRD had negative charge after second revolution. These observations suggest that the polarity of static electrification due to asymmetric rubbing are sensitive to the condition of the surface. For $\mathrm{PE}$, on one day SRD tended to have positive charge, and on the other day negative charge. For PMMA and PC, both case of polarity were observed and on one day the polarity was biased to one disk as shown in Fig.3. In one series of observation for POM, although the observed amplitude is small due to escape of charge, SRD seemed to be negatively charged for the ratio 2 of the rotating 
speeds and positively charged for the ratio 5,10 . Note that this does not necessarily suggest that the origin of the alternation is the ratio of speeds. Further careful investigation would clarify this point. As presented here, there were various phenomena and it is presently difficult to predict even the sign of charge separation in all cases.

Next, consider the symmetric rolling friction of identical materials. Although there was no apparent reason for the separation of charge in this case, the separation was observed as shown in Fig.4, which shows surface voltage after two identical samples went into contact and made 0.9 round symmetrical roll. The values of voltage at corresponding contacted location of two samples are found to be of the same magnitude with the opposite sign with each other. Here the origin of this charge separation is unclear but some unknown difference of the surfaces would give rise to the separation. After many revolutions, the separation did not disappear. One may hope that the charge should be neutralized when two surfaces holding inverse charge are merged very slowly, but it is not observed at least in $0.012 \mathrm{rpm}$ rolling condition. Typically, the observed values of the surface voltage after symmetric rolling of identical materials were not so high compared to the cases between different materials or the cases of asymmetric rubbing.

\section{SUMMARY}

In this paper, we have investigated triboelectrification of plastic materials controlling rubbing speed. Electrostatic series was obtained for contact electrification between different materials. Then electrification between same kind of materials was examined and it was observed that static electrification due to asymmetric rubbing actually occurred. In most cases, the larger magnitude of the surface voltage was observed on the slower rotating disk (SRD) and the sign of the voltage depended on materials. For some materials, SRD tended to have positive charge, and for some other materials to have negative charge, i.e. there were two types of charge separation. In the case that SRD have positive charge, it might be regarded as a result of thermal diffusion of electron due to a temperature gradient, because the temperature of the SRD should be higher than that of the faster rotating disk. This idea, however, cannot be applied to the cases in which SRD are negatively charged. It is yet unknown what kinds of carrier make these charge separations and there might be two or more mechanisms for the separation. It should be investigated in future what determines the sign of the separation. In addition, there were many exceptional observations and it was not easy to predict the sign of the charge appeared on the surface in all cases. These observations suggest static electrification due to asymmetric rubbing is sensitive to the condition of the surface. Further, in case of symmetric rolling, measurable opposite charge arose on each disk surface, whose origin might be some unknown difference between the
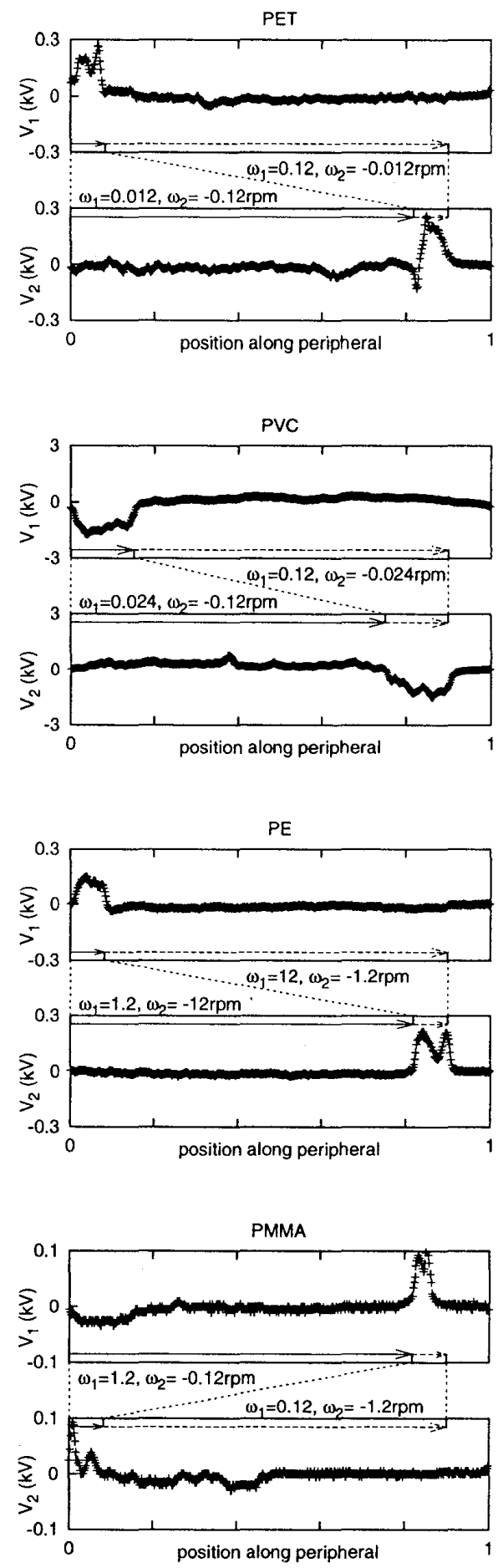

FIG. 3: Electrification due to asymmetric rubbing of identical materials. (PET,PVC,PE,PMMA). Horizontal axes are positions along the peripherals in the same manner as in Fig. 2 . 

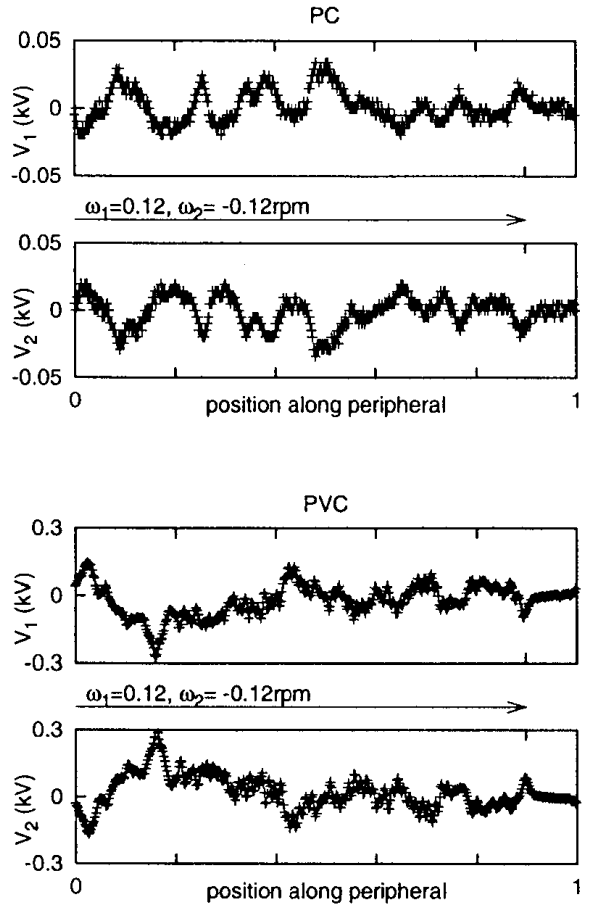

FIG. 4: Electrification due to symmetric rolling of identical materials (PC, PVC). In these experiments, samples were symmetrically rolled with $\omega_{1}=-\omega_{2}=0.12 \mathrm{rpm}$. Horizontal axes are positions along the peripherals in the same manner as in Fig. 2.

surfaces. This might suggest some instability mechanism for the separation of charge.

The variety of phenomena in triboelectrification would be caused by "the state of the surface": in part it might be described by the roughness, the attachment of worn material or dust, the scratch on the surface, the intertwist structure of the polymer at the surface, etc. The phenomena would be sensitive to these state of the surface. There might be multiple mechanisms of charge separation, which make appearance of phenomena more complicated. The effects of memory and wear would be also reasons why the observed phenomena shows much variety and it is still far from predictable what occurs after many kinds of contacts. The observations presented here are just a first step toward understanding of static electrification and further studies will be continued to clarify the mechanism and also to find some relation between the electrification and the control parameters (the rolling speed, normal force, etc.) .

One of the difficulties encountered in this experiment was the preparation of the samples. We still do not know to what precision and cleanliness the surface should be prepared to ensure no charge separation in symmetrical contact of identical materials. Further, once the surface is contacted, the surface condition should vary and some extent of wear should occur. There might be some mechanism by which slight difference of charge distribution between two surfaces grows up and the charge separation might result from some instability of the separation process.

\section{ACKNOWLEDGMENT}

The authors acknowledge H. Miyagi and Y. Shimokawa for their support of building the experimental apparatus. One of the authors (TSK) greatly appreciates the advices for our system from K. Hiratsuka and T. Yoshida. Authors also thank $\mathrm{H}$. Tasaki for his encouragement and critical reading of this manuscript.
(1) W. J. Brennan, J. Lowell, G.W. Fellows and M. P. W. Wilson, J. Phys. D, Appl. Phys. 28 (1995) 2349.

[2] K. Ohara, J. Electrostatics 4 (1978) 233.

[3] I. Shinohara, Y. Fujio, H. Anzai and S. Endo, J. Electrostatics 2 (1976) 99.

[4] R. Elsdon and F. R. G. Mitchell, J. Phys. D. Appl. Phys.
9 (1976) 1445.

[5] H. R. Harper, Proc. R. Soc. A 231 (1955) 388.

[6] P. S. H. Henry, J. Appl. Phys. Suppl. 2 (1953) S31.

[7] J. Lowell and A. C. Rose-Innes, Adv. Phys. 29 (1980) 1009. 